\title{
Empowering Elderly End-Users for Ambient Programming: The Tangible Way
}

\author{
Johan Criel, Marjan Geerts, Laurence Claeys and Fahim Kawsar \\ Bell Laboratories, Alcatel-Lucent Bell N.V., Belgium \\ \{johan.criel, marjan.geerts, laurence.claeys, \\ fahim.kawsar\}@alcatel-lucent.com
}

\begin{abstract}
This paper presents a novel tangible interaction technique leveraging Near Field Communication (NFC) enabled Magnetic Cards to empower elderly end-users in programming their pervasive computing environment. Following a user-centric design approach through cultural probes, we have explored the design space of possible programming opportunities for elderly users. Accordingly we have identified interaction techniques and candidate artefacts that could offer this user group a seamless programming experience for augmenting their ambient environment with software driven personalized behavior. Three aspects of programming - behavior generation, modification and (de) activation are addressed using two tangible user interfaces - i) NFC cards for smart behavior creation and modification and ii) a digital memo board as a placeholder for (de) activation of intelligent behavior. A qualitative feasibility study suggests that the proposed approach is simple, comprehensive and has the potential to be easily incorporated in the everyday routines of the elderly end-users.
\end{abstract}

\section{Introduction}

Pervasive computing has instigated a transformation of our environment into an interactive information sphere spread across time and space by combining sensing and software services with physical world. This essentially creates novel design opportunities for everyday artefacts - so called smart objects, as their behaviour are determined by software. This affords us to build imaginative new forms of interaction and functionalities that dramatically enhances their well-established features. While the technological building blocks for realizing such programmable physical space is converging there is a clear need to shift the focus onto end-users. It is essential to empower end-users with an appropriate capacity to program this interactive physical space populated with a range of smart objects and enable them to unleash computing enabled creativity capitalizing the possibilities offered by pervasive computing. Contemporary work has argued that it is very crucial to bring end-users into the center of control and management of pervasive computing environment $[2,13-15]$ as it is the people who occupy those environments and have the best knowledge about how their physical and computational environments should respond to their activities. Earlier research also demonstrated the prospect of involving end-users in creative engagement and its implication in raising end-users adoption and understanding of pervasive computing systems. For instance, with the SpeakEasy platform end-users can search and compose semantically compatible appliances [7] and the Jigsaw Editor enables end-users to assemble jigsaw pieces 
representing pervasive services and smart artefacts to construct simple programs (e.g., connecting a doorbell to a camera for taking a photo shot when someone rings the bell) [1]. Also, a number of projects explored singular screen centric rule and recognition tools [4-6] to support end-users in personalising simple tasks, e.g., turning on/off lights contextually, etc.

However, unfortunately these solutions are not suitable for one class of citizen that represents a large population of our society : the seniors. In fact the diffusion of pervasive technologies in the home of senior citizens happens very slowly. Two primary reasons are: i) the technophobia of part of the elderly population and consequent anxiety towards technology usage and ii) the design principles of the application that fails to address elderly centric needs. [12]. The technology demand as well as interaction experience of senior citizens are fundamentally different than regular users, and an aspect like programming obviously puts significant burden on the designers. A classical evidence is that none of the projects mentioned above have addressed elderly-centric needs in their design. In this paper, we address this particular challenging issue of enabling elderly users to experience pervasive computing environment as well as program the environment to meet their customised needs.

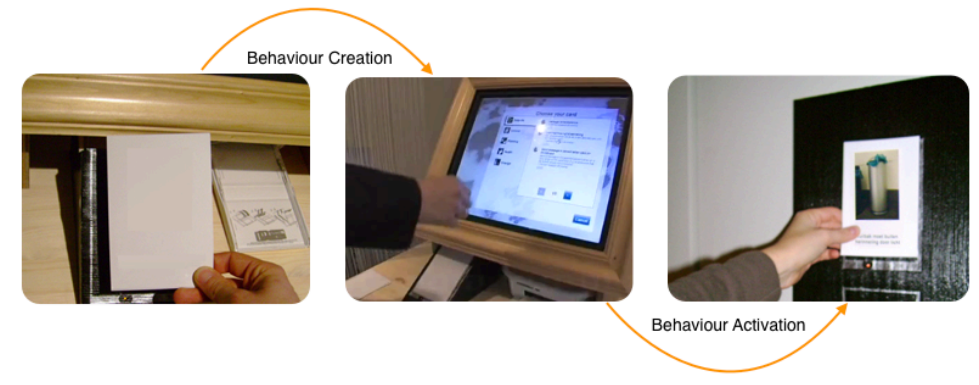

Fig. 1. NFC-enabled magnetic cards are used to create customised behaviour from a range of templates and by setting appropriate conditions. This behaviour can be activated and deactivated by placing/removing the card onto/from a digital memo board.

Methodologically, we approach this in a top down fashion by taking a look at the life world of seniors and listening to the seniors themselves to have a profound understanding of what seniors want to do, how they want to do it in their ambient environment and with what granularity. This is unlike existing work in pervasive computing where tools were developed and given to end-users to configure their everyday lives in a predetermined fashion without addressing their actual needs. This user centric approach gives us a deeper insight on elderly end-users requirement and affords us to design a solution that fits their need. By exploring the design space emerged from our insight, we have identified three aspects of programming that elderly users would like to be involved in : smart behaviour creation, modification and (de)activation. By technology probing, we converted these needs into a tangible interaction experience using simple cards and a memo board commonly found in our home. In particular, NFC-enabled magnetic cards allow elderly users to create personalized behaviour for their ambient environment and a digital memo board acts as a place holder for active behaviour ensuring the metaphoric resemblance of a memo board and post cards (Fig 1). Examples 
of such custom generated behaviours of ambient environment are : Setting reminder for grandma to put the garbage outside on Wednesday by switching on a lamp above the garbage can; Sending awareness SMS to caregiver when grandma opens the curtains in the morning, to relay that grandma woke up, etc. In this paper, we present the design space analysis through cultural probes to understand such needs and possible opportunities for translating these needs into technology constructs. This is followed by the technical description of the tangible interfaces and interaction techniques emerged from our analysis. We also present a qualitative in-situ assessment of our proposed approach to highlight its strength and caveats.

The primary contribution of this paper is twofold - firstly, a design space reflection exposing the technology demands for creative engagement of elderly end-users and secondly a concrete design prototype of a tangible interfaces and corresponding interaction techniques to empower elderly end-users in programming the ambient environment.

\section{Understanding the Design Space through Cultural Probes}

End users are not programmers in the traditional sense. It is important to apprehend what end-users currently do, what they want to do as well as what technology can address those needs. A concrete understanding of these issues naturally leads to a technical solution that is more close to end user needs. In the context of senior citizens this is very crucial as their familiarity with technology as well as technical skills to interact with technology is relatively poor due to their age and possible age related diseases. Consequently we began our research by looking and listening to the seniors themselves. The contextual investigation we performed can be defined as an "applied ethnography" using two different methods: cultural probing and interviewing. 16 elderly persons who live alone (without partner) participated in the study - 8 senior persons were above 75 years old and the rest were above 60 years old. In addition, 8 caregivers for the participants with early dementia also participated in the study which allowed us to understand the technology demands of elderly that can be supported by their caregivers. All of the participants speak the Dutch language and live in the West-Flemish part of Belgium. The objective of their participation in the study was to formulate a qualitative understanding of the following three questions:

- What are the routines of senior citizens (with/without early dementia) who live alone?

- How do senior citizens who live alone adapt their in-home life?

- How do senior citizens with early dementia who live alone adapt their in-home life with the help of their caregivers?

The first phase of the study was carried out through cultural probing. The cultural probe that is used for ethnographic research, as in our case, is a kit that holds a variety of materials that trigger people to do individual research in their own lives $[8,9]$. It is designed to engage participants in a study, and to stimulate them to response to the exercises that they find in the kit. The output of the participants is partly a source of inspiration to those involved in research and design i.e. ethnographers, designers and engineers, and partly the departure point for a qualitative interview. The probe (Fig. 2) we used consists of a box full of nice items: a diary, a contact bulb, a helper-frame, a sheet to draw a home plan, stickers, a photo camera and the usual probe stuff - colored pens, a card with contact information, a notebook and some candy. 

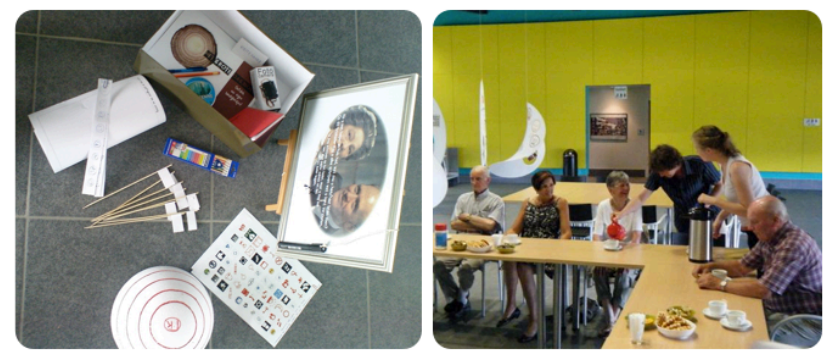

Fig. 2. The Cultural Probe Kit (left) and the Interview Session (right).

The dairy forms the guideline of the probe. From day to day, the diary guided the participant through exercises on a certain domain. The domains were based on nine life fields including - day time spending, mental health, life style, life questions, physical health, education, social contacts, leisure time and residence. The elderly got three weeks time to use the probe kit before it was returned to the researchers. During that period, the researchers tried to stimulate the participant by sending a couple of postcards to his/her home address. On these post cards, additional questions were written, and each of them could be pasted inside the diary. The second phase of the study involved face-to-face interviewing with every participant. All interviews were coded (in total 994 codes were assigned) in the analysis software MAXQDA, analysed and discussed by the researchers and communicated to the rest of the teams.

\subsection{Implications for Design}

The investigation allowed us to gain a deeper understanding of the technology practices and demands of elderly end users. Considering the context of this paper we will limit the discussion in this section around end-user programming only. The detailed findings of the study is reported in other forums $[10,11]$. In the following we report the implications of the study that lead us to formulate the design principles of our eventual solution.

Plethora of Needs: The elderly citizen, specially with dementia has a greater need for assistance even for rudimentary activities (e.g., brushing teeth, taking medicines, putting garbage outside, turning lamps on while reading, etc.) that seem obvious for other age groups. The three most prominent needs can be generalised as situated assistance, and support for reminding and notifying caregiver. The take away note is the fact that - it is imperative to empower seniors (and their caregivers) to generate customised solutions to address at least these needs.

Ownership: It was evident from our study that seniors want to have full control of their activities. They usually take extreme pride of little things they do, so-called "life hacks" to be in control of their lives and these tweaks also give them the feeling of ownership of the consequent effects in their lives.

Object Centric Life: Seniors in general structure their life by time. If they get dementia caregivers takes over the structuring of their life around some objects, so a change from 
time-centric life to object-centric life takes place. This essentially exposes the necessity for the instrumentation of everyday objects offering situated assistance.

Intelligibility - Control and Consistent Feedback: It is absolutely necessary to give full control to the seniors taking into consideration their capacity of cognitive and motor skills. Therefore, it is essential provide consistent, large and visual feedback that are recognisable to seniors so that they know what is happening around them and how they can control it. This is specially important for seniors suffering from dementia.

Simplicity: It is obvious that technology for seniors has to be straight-forward and should offer very basic functions that meet their needs. It is necessary to keep the balance between simplicity and rich functionality by carefully designing necessary features and keeping them relatively open to encourage endusers' active participation.

Tangibility and Mechanical Experience: It is essential to provide seniors with technology that offers a tangible and mechanical experience. Although current research is geared towards making tiny and invisible interfaces, for this class of user group it is still important to keep the tangibility and mechanical quality of experience. Pressing a button or turning a knob etc. is preferred over touch screen or other context driven triggers (e.g., position, location, etc.).

Memo Board as the Control Panel: Seniors organise their indoor and outdoor activities using a lot of memos and sticky notes pasted on the memo board or other flat surfaces (e.g., doors, refrigerator surface, etc.) as they need constant reminders of things to be done. Such practice gives us a justified choice to use memo board as the primary workplace where seniors can configure their technological assistance.

To summarise, the study clearly exposed the fact that - it is essential to empower elderly citizen with technology that allow them to create customised object centric solutions to address simple needs and get situated assistances in their lives. Accordingly, an imperative aspect of technology for ambient assisted living is involving elderly citizen closely with the technology for an intimate as well as vital interaction experience.

End user programming in its simplest form thus turns out to be a crucial component for ambient assistance. However, technology has to be simple, intelligible, personalized with large visual feedback and should give the ownership to seniors. Tangible interface has been identified as the major form of interaction due to its mechanical quality of

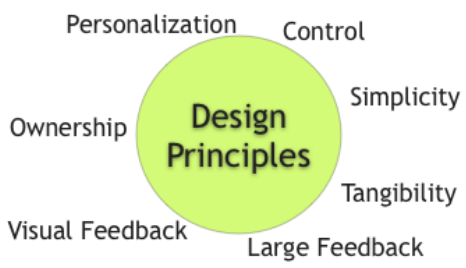

Fig. 3. Design Principles to engage Elderly Endusers in Creative Engagement.

experience. In addition, we have identified that the memo board commonly found in elderly homes is an ideal object that can be used as the workplace of seniors for creating their personalized technology solutions. In the next section, we discuss the technical translations of these principles into concrete technology prototypes.

\section{Tangible Interface and Programming Techniques}

Following the implications of the study discussed in the previous section, it is evident that to involve elderly endusers in creative engagement we need to design the interface and corresponding interaction technique as simple as possible. We address this by 
identifying two common objects that can be manipulated in elderly home: sticky notes and memo boards, and augmenting them to enable simple tangible programming experience. The basic approach of our solution is to use NFC-enabled magnetic cards to create custom tailored behaviour and to place them on a digital memo board to activate or deactivate the behaviour resembling the metaphor of a sticky note pasted on the memo board.

\subsection{Tangible Interface}

The user interface for supporting tangible programming is composed of three components designed following the principles mentioned earlier.

- NFC enabled Magnetic Cards: These cards are made of magnetic photo paper with a NFC chip attached on the back side of the paper (Fig. 4(a)). Once a behaviour is generated (discussed next) a personalized photo image sticker is printed that can be pasted on the front side of the card for large, visual and recognisable representation of the behaviour .

- Touch Screen Terminal for Behaviour Generation: A touch screen terminal is augmented with a NFC reader (Fig. 4(b)). This terminal provides support for creating and modifying custom tailored behaviour for ambient environment. In addition, a printer attached to this terminal allows printing personalized stickers that can be attached to the cards.

- Digital Memo Board:The digital memo board is equipped with five NFC readers on the back (Fig. 4(c)). The locations of these readers are displayed in the form of boxes on the front, to make it easier for the user to position the cards in the right way. The memo board is covered with a layer of small, round magnets to hold the cards. Below each box on the memo board, a Light Emitting Diode (LED) is placed to give visual feedback. This LED is illuminated when a card is placed on the board ensuring that the behaviour is correctly recognised and activated. The digital memo board can be mounted to the wall in the home on eye level of the user.

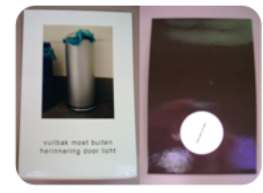

(a) NFC enabled Magnetic Cards

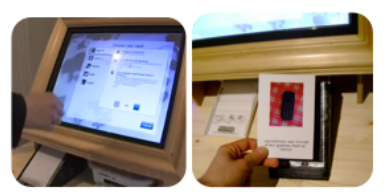

(b) Touch Screen Terminal

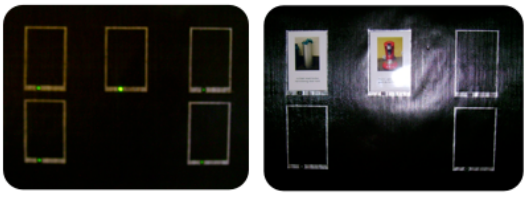

(c) Digital Memo Board

Fig. 4. Tangible Interface for Tangible Programming

An underlying infrastructure called CAEMP supports the tracking, interpretation as well as the consequent spontaneous federation of smart objects to facilitate these custom generated behaviour [16].

\subsection{Tangible Programming Techniques}

Three aspects of programming are addressed using the tangible interface presented above, these are - smart behaviour creation, smart behaviour modification and finally (de) activation of the behaviour. 
Tangible Interaction for Smart Behaviour Creation: New smart behavior is created using the magnetic cards and the touch screen terminal (Fig. 5). The first step is placing a blank card on the touch screen terminal. Immediately, the smart behaviour creation environment starts up on the touch screen. The user can select a smart behavior template from the available template collection. An example of such a template is the Show Media Template that can be used to show assistive media on a screen if a person touches a specific smart object. The user can fill in the template with a selection of users, conditions, objects and actions. When the user is satisfied with the defined smart behavior, a description of the behaviour along with a custom defined representative image can be printed and can be attached to the card.

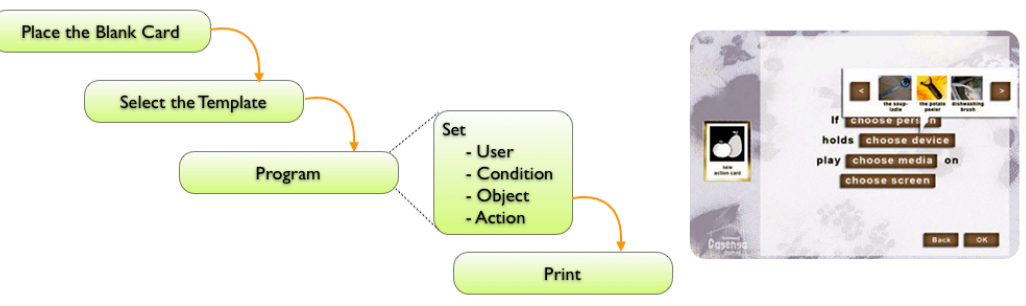

Fig. 5. Tangible Interaction for Smart Behaviour Creation

Tangible Interaction for Smart Behaviour Modification: Any existing smart behaviour can be modified or even deleted, using the touch screen terminal of the creation environment. To modify the smart behaviour, the representative card is placed on the terminal and immediately the programming environment is shown on the touch screen that end-users can manipulate (selection of users, conditions, objects and actions) to alter the old behaviour (Fig.6). After confirmation, the old behaviour is replaced by the newly modified behaviour on the card.
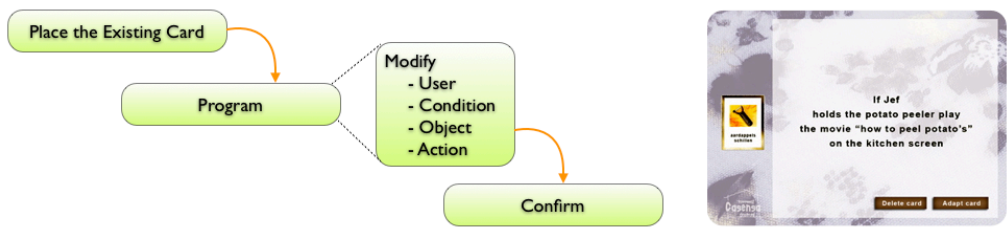

Fig. 6. Tangible Interaction for Smart Behaviour Modification

Tangible Interaction for (De) Activation of the Smart Behavior: To activate a new behaviour in the ambient environment, all users need to do is to place the card on the augmented digital memo board. Whenever a card is positioned in one of the frames on the memo board, the accompanying behaviour is active (Fig.7). The user can check if the card is positioned well, through the LED that is blinking under the frame. By simply removing the card from the memo board the user can deactivate its embedded smart behaviour. 


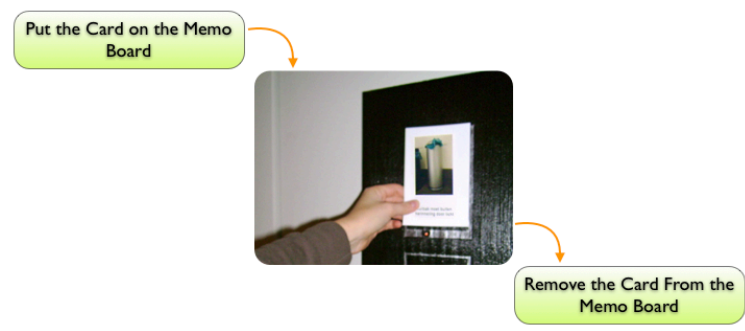

Fig. 7. Tangible Interaction for Smart Behaviour Activation and Deactivation

As shown above, the combination of personalized magnetic cards and digital memo board interaction allows us to maintain the tangibility and simplicity of the programming experience. Additionally, particular emphasis is given to ensure that elderly endusers are put in the centre of control for the consequent behaviour as they can personalize, activate and deactivate these behaviours in a straight-forward fashion. Furthermore, large, visual and recognisable behaviour representation and consistent feedback through LEDs made sure that the interface and interaction experience meet the design principles. In the next section, we present an in-situ assessment of this tangible interface and corresponding programming techniques.

\section{Feasibility Study in the Living Lab}

The prototype tangible interface along with its underlying infrastructure [16] was installed in a living lab apartment that was furnished with smart objects, like a smart alarm clock, a smart toothbrush, a smart coffee machine, a smart garbage can, a digital photo frame in the living room and a digital screen in the kitchen. Both the touch screen terminal and the digital memo board were placed in that apartment ${ }^{1}$. A range of behaviour templates were available integrating these smart objects, for example : Remind grandma to put the garbage outside on certain day by switching on a lamp above the garbage can, Start the television every day on a certain channel to remind grandma that her favourite television program is starting, Display pictures of grandmas wedding on the digital photo frame on every wedding anniversary, etc. To qualitatively assess our prototype, 8 elderly participants (age range above 60 years) and 2 care givers (all of them participated in the cultural probe session) were invited to visit the apartment. They were given basic information about the prototype and its objective, and were asked to perform certain tasks involving creation, modification and (de)activation of behaviours and then explain it to another participant. The test was done in a role-play format. At the end, the participants were interviewed and had to fill in a short survey. This study focused on the experience and usability of the proposed design. Our qualitative assessment of their performance and follow up interview clearly showed that all the elderly citizens were able to grasp the concept completely and found the process of programming straight forward. However, it was indicated that the behaviour creation phase could be relayed to the care givers, as sometimes elderly citizens got confused what can

\footnotetext{
${ }^{1}$ Please have a look at http://bit.ly/casensa to experience our living lab, tangible interface and programming techniques as well as running use cases.
} 
be done with the available objects. Once a behaviour is created, it was straight-forward for them to perform the other steps - modification, activation, etc. The large, visual feedback through picture and LEDs were found to be very crucial for their understanding. They also mentioned that these feedback gave them a sense of awareness and control of the environment. The major caveats of the system was the user interface of the touch screen terminal - the creation environment, as some screens' layout confused them due to the misplacement of buttons and corresponding pictures. In the later versions of the prototype we have addressed these issues.

\section{Related Work and Conclusion}

End User programming in pervasive computing environment is emerging as a hot area of research with the convergence of the technological building blocks of pervasive computing. Early seminal work on this area include Jigsaw Editor [1] that uses puzzle metaphor and allows non-expert users to configure pervasive services intuitively by assembling available components, and application of SpeakEasy computing platform that enables end-users to compose pervasive services spontaneously. Other work focused on the end user deployment activities and demonstrated that end-users understand the semantic association of physical devices and digital software [2]. Kawsar et al. presented a similar approach like ours using RFID Cards equipped with predefined custom behaviour. However, their work did not address the creation and modification aspects. In addition, we argue that our Digital Memo Board metaphor is more natural than their dedicated deployment tool [3]. Some work also addressed support for end-users personalization through rule or recognition based tools using graphical user interface (GUI). Rule based tools like iCAP [4], Alfred [6] provide visual tools, or sound macros to the end-users to define conditional rules based on the context to connect input and output events. Similarly recognition tools, or more formally Programming by Demonstration systems like CAPpella [5] use machine learning techniques to allow end-users to associate rules with real world events. These approaches are valid for rapid prototyping. However, we do not consider they are suitable for non-technical users, especially the elderly user group. Furthermore, these tools are designed for supporting very simple end-user tasks, such as turning on/off a device, loading presentation files, etc. These solutions primarily focused on facilitating the initial configuration tasks only, and it is not clear how well they can support custom tailored behaviour generation, modification and (de) activation as user requirements and environment change. In our proposed approach, particular emphasis is given to a design approach that radically simplifies these activities though use of simple cards yet enabling the generation of personalized behaviour.

To conclude, we have presented a novel tangible programming interface and corresponding interaction technique specifically aimed at involving elderly citizens in creative engagement of programming ambient environment in a straight-forward fashion. Following a top-down user centric approach, we have presented an applied ethnographic study that helped us to formulate the design principles and corresponding technology prototypes . We also touched upon the qualitative assessment of our approach. We hope that, our technology prototype as well as the implications emerged from our multi phase studies will contribute in shaping the future of ambient assisted living technologies significantly. 
Acknowledgement: The work reported in this paper was performed in the context of the ITEA2 AmIE project - funded by the Flemish IWT.

\section{References}

1. Humble, J., Crabtree, A., Hemmings, T., Akesson, K. P., Koleva, B., Rodden, T., and Hansson, P. (2003). Playing with your bits: User composition of ubiquitous domestic environments. In 5th International Conference on Ubiquitous Computing (Ubicomp 2003).

2. Beckmann, C., Consolvo, S., and LaMarca, A. (2004). Some assembly required: Supporting end-user sensor installation in domestic ubiquitous computing environments. In 6th international conference on Ubiquitous computing (UbiComp 2004), pages 107-124.

3. Kawsar, F., Fujinami, K. and Nakajima, T., (2008) Deploy Spontaneously: Supporting EndUsers in Building and Enhancing a Smart Home; The 10th International Conference on Ubiquitous Computing (Ubicomp 2008), pages 282-291.

4. Dey, A. K., Shon, T., Streng, S., and Kodama, J. (2006). iCap: Interactive prototyping of context-aware applications. In 4th International Conference on Pervasive Computing (Pervasive 2006), pages 254-271.

5. Dey, A. K., Hamid, R., Beckmann, C., Li, I., and Hsu, D. (2004). a CAPpella: Programming by demonstration of context-aware applications. In ACM Conference on Human Factors in Computing Systems (CHI 2004), pages 33-40.

6. Gajos, K., Fox, H., and Shrobe, H. (2002). End user empowerment in human centered pervasive computing. In International Conference on Pervasive Computing (Pervasive 2002).

7. Edwards, W. K., Newman, M., Sedivy, J., Smith, T., and Izadi, S. (2002). Challenge: Recombinant computing and the speakeasy approach. In 8th Annual International Conference on Mobile Computing and Networking (MobiCom 2002), pages 279-286.

8. Graham, C., Rouncefield, M, Gibbs, M., Vetere, F and Cheverest, K.: How Probes Work. Proceedings of the Conference of the Computer-Human Interaction Special Interest Group (CHISIG) of Australia on Computer-Human Interaction: Design, activities, artifacts and environments. November 2007, ACM Publisher (2007)

9. Gaver, B., Dunne, T. and Pacenti, E.: Design: Cultural Probes. Interactions, 6(1), 1999

10. Geerts M., Criel J., Claeys L., Godon M., Dieryckx, L., and Deboutte. P., : Let the Seniors Hack! Finding daily life practices and hacks of elderly using cultural probes, Workshop Capturing Ambient Assisted Living Needs, Ambient Intelligence Conference '08, Germany.

11. Geerts M., Claeys L., Criel J., et al. Seniors re-engineering context-aware applications. The casensa research process. In: proceedings of AmI-09 Conference. Salzburg, 2009.

12. Claeys ,L. and Criel, J.: Context aware computing: Future living as a social application. In: Withworth, B., Demoor, A. (eds.) Handbook of research on socio-technical design, pp.779793. IGIGlobal, London (2008)

13. Rodden, T. and Benford, S. (2003). The evolution of buildings and implications for the design of ubiquitous domestic environments. In ACM Conference on Human Factors in Computing Systems (CHI 2003), pages 9-16.

14. O'Brien, J., Rodden, T., and Hughes, M. R. J. (1999). At home with the technology: an ethnographic study of a set-top-box trial. ACM Transactions on Computer Human Interaction, 6(3), pages 282-308.

15. Edwards, W. K. and Grinter, R. (2001). At home with ubiquitous computing: Seven challenges. In 3rd International Conference on Ubiquitous Computing (UbiComp 2001).

16. Criel, J., Claeys, L., and Trappeniers, L. Deconstructing Casensa: The CAEMP ContextAware Empowering Platform: , Bell Labs Technical Journal Issue, Vol. 16, No. 1, 2011 